\title{
Azimuthal distributions of radial momentum and velocity in relativistic heavy ion collisions
}

\author{
Lin $\mathrm{Li}^{1}{ }^{1} \mathrm{Na} \mathrm{Li},{ }^{2}$ and Yuanfang $\mathrm{Wu}^{1,3,4}$ \\ ${ }^{1}$ Institute of Particle Physics, Hua-Zhong Normal University, Wuhan 430079, China \\ ${ }^{2}$ Hua-zhong University of Science and Technology, 4300\%4, China \\ ${ }^{3}$ Brookhaven National Laboratory, Upton, NY 11973, U.S.A. \\ ${ }^{4}$ Key Laboratory of Quark \& Lepton Physics (Huazhong Normal University), Ministry of Education, China
}

\begin{abstract}
Azimuthal distributions of radial (transverse) momentum, mean radial momentum, and mean radial velocity of final state particles are suggested for relativistic heavy ion collisions. Using transport model AMPT with string melting, these distributions for $\mathrm{Au}+\mathrm{Au}$ collisions at $200 \mathrm{GeV}$ are presented and studied. It is demonstrated that the distribution of total radial momentum is more sensitive to the anisotropic expansion, as the anisotropies of final state particles and their associated transverse momentums are both counted in the measure. The mean radial velocity distribution is compared with the radial flow velocity. The thermal motion contributes an isotropic constant to mean radial velocity.
\end{abstract}

PACS numbers:

\section{INTRODUCTION}

One of the main goals of current relativistic heavy ion collisions is to understand the properties of a new formed matter - the quark-gluon plasma (QGP) 1]. It is well known that one important character of the formed matter is anisotropic collective flow. In non-central collisions, the overlap area of two incident nuclei is an almond shape in the transverse coordinate plane [2]. This initial geometric asymmetry leads larger density gradient along the short axis. It in turn pushes the formed system to expand anisotropically, i.e., large collective flow velocity in short side direction, which is perpendicular to the anisotropy in coordinate space. Therefore, the measure of anisotropic distribution of final state particles should provide valuable information of the system evolution [2, 3].

Conventionally, the azimuthal distribution of multiplicity of final state particles is presented. Its anisotropy is quantified by the coefficients of Fourier expansion of the distribution [4]

$$
\frac{d N}{d \phi} \propto 1+\sum_{n=1}^{\infty} 2 v_{n}(N) \cos (n \phi)
$$

where $\phi$ is the azimuthal angle between the transverse momentum of the particle and the reaction plane. The Fourier coefficients is evaluated by,

$$
v_{n}(N)=\langle\cos (n \phi)\rangle,
$$

where $\langle\ldots\rangle$ is an average over all particles in all events, and $v_{n}(N)$ refers to the anisotropy coefficient of azimuthal multiplicity distribution. The second harmonic coefficient $v_{2}(N)$ is the so-called elliptic flow parameter. It presents the anisotropy of the colliding system and has the biggest ellipticity at high energy heavy ion collisions [5, 6]. Besides, the azimuthal asymmetry distribution of energy loss and its coefficients of Fourier expansion are also studied [7].
However, the multiplicity distribution only counts the number of particles emission in a certain azimuthal angle. The expansion of the system results in not only the anisotropy of multiplicity distribution but also their associate radial (transverse) momentum. The total radial momenta at a given azimuthal angle is the combination of them. Therefore, the azimuthal distribution of radial momentum should be a more sensitive measure of the anisotropic expansion, which has not been directly explored before.

In addition to the radial momentum, the radial flow velocity is another interesting and important quantity. It directly relates to the equation of state [8] and shear viscous interactions. For an ideal flow, the radial flow velocity is isotropy. While, if there are shear interactions, the radial flow velocity will be different from layer to layer. In hydrodynamics, the shear viscous interactions are supposed to be proportional to the gradient of flow velocity [9]. The proportional constant is defined as shear viscosity. The gradient of radial flow velocity along the azimuthal direction is directly related to the shear viscous interactions.

Theoretically, the radial flow velocity is a parameter in model calculations. It is usually obtained by fitting the spectrum of transverse momentum [10]. Recently, it is further suggested to extract the radial flow velocity from photon and dilepton spectrum [11].

Experimentally, only the radial velocity of final state particles $(\vec{v})$ is measurable. It should be a combination of velocities of flow $\left(\vec{v}_{\text {flow }}\right)$ and the random thermal motion $\left(\vec{v}_{\mathrm{th}}\right)$ [12]. How to abstract the random thermal motion from the radial velocity of final state particles and get the radial flow velocity are not clear. This is why the radial velocity of final state particles has not been explored in a long period. It is interesting to see how the radial velocity of final state particle relates to the radial flow velocity. Therefore, we further suggest the measurement of the azimuthal distribution of mean radial velocity of final state particles. 
In the second session of the paper, we will give the definitions of suggested azimuthal distributions of radial momentum and velocity of final state particles, and the corresponding anisotropic parameters. In the third session, using the samples generated by AMPT with string melting model, we show the azimuthal distributions of radial momentum and the centrality dependence of its anisotropic parameters. The results are compared with those of the corresponding azimuthal multiplicity distribution. In the fourth session, the azimuthal distributions of mean radial velocity at different centralities are presented, and compared with those given by the anisotropic blast-wave model [13 15]. Finally, the summary and conclusions are presented.

\section{AZIMUTHAL DISTRIBUTIONS OF RADIAL MOMENTUM AND VELOCITY}

As indicated, the initial anisotropy in coordinate space in non-central collisions makes the formed system expand in a perpendicular almond shape in momentum space. The final state particles move outward anisotropically. Both the particle density and the associated momentum behaves anisotropically during the expansion. The distribution of total transverse momentum at the different azimuthal directions should be a good measurement for both of these two effects. The total transverse momentum in the $m$ th azimuthal bin can be defined as

$$
\left\langle P_{\mathrm{t}}\left(\phi_{m}\right)\right\rangle=\frac{1}{N_{\text {event }}} \sum_{j=1}^{N_{\text {event }}}\left(\sum_{i=1}^{N_{m}} p_{\mathrm{t}, i}\left(\phi_{m}\right)\right) .
$$

where $p_{\mathrm{t}, i}$ is the transverse momentum of the $i$ th particle, $N_{m}$ is the total number of particles, and $\langle\ldots\rangle$ denotes the average over all events.

In order to see the contributions of radial momentum in particular, the mean radial momentum in the $m$ th azimuthal bin can be defined accordingly as,

$$
\left\langle\left\langle p_{\mathrm{t}}\left(\phi_{m}\right)\right\rangle\right\rangle=\frac{1}{N_{\text {event }}} \sum_{j=1}^{N_{\text {event }}}\left(\frac{1}{N_{m}} \sum_{i=1}^{N_{m}} p_{\mathrm{t}, i}\left(\phi_{m}\right)\right) .
$$

Here, the averages $\langle\langle\ldots\rangle\rangle$ are over all particles in $m$ th angle bin and all events. It records only the contributions from the transverse momentum of final particles, the multiplicity effect is canceled by the average over all particles.

The anisotropic parameters of all those azimuthal distributions can be directly obtained from their Fourier expansions, respectively,

$$
\frac{d\left\langle P_{\mathrm{t}}\right\rangle}{d \phi} \propto 1+\sum_{n=1}^{\infty} 2 v_{n}\left(\left\langle P_{\mathrm{t}}\right\rangle\right) \cos (n \phi)
$$

and

$$
\frac{d\left\langle\left\langle p_{\mathrm{t}}\right\rangle\right\rangle}{d \phi} \propto 1+\sum_{n=1}^{\infty} 2 v_{n}\left(\left\langle\left\langle p_{\mathrm{t}}\right\rangle\right\rangle\right) \cos (n \phi)
$$

$\frac{d\left\langle P_{\mathrm{t}}\right\rangle}{d \phi}$ and $\frac{d\left\langle\left\langle p_{\mathrm{t}}\right\rangle\right\rangle}{d \phi}$ are the azimuthal distribution functions of total radial momentum and mean radial momentum. $v_{n}\left(\left\langle P_{\mathrm{t}}\right\rangle\right)$ and $v_{n}\left(\left\langle\left\langle p_{\mathrm{t}}\right\rangle\right\rangle\right)$ are their anisotropic parameters, respectively.

Considering the relativistic effect, the transverse (radial) velocity of the $i$ th particle can be written as,

$$
v_{t, i}=\frac{p_{t, i}}{m_{\mathrm{t}}}=\frac{p_{t, i}}{\sqrt{m_{0, i}^{2}+p_{t, i}^{2}}},
$$

where $p_{t, i}$ and $m_{t, i}$ are the transverse momentum and mass of the $i$ th particle, respectively. $m_{0, i}$ is the mass of $i$ th particle in rest frame. The radial velocity fluctuates from particle to particle. In a given azimuthal direction, the mean radial velocity can be considered as a good approximation. Analogously, the azimuthal distribution of mean radial velocity can be defined as

$$
\left\langle\left\langle v_{\mathrm{t}}\left(\phi_{m}\right)\right\rangle\right\rangle=\frac{1}{N_{\text {event }}} \sum_{j=1}^{N_{\text {event }}}\left(\frac{1}{N_{m}} \sum_{i=1}^{N_{m}} v_{\mathrm{t}, i}\left(\phi_{m}\right)\right),
$$

Here, the average is over all the particles in $m$ th bin and events.

The behavior of those suggested observables should provide more information in anisotropic expansion. In the following, as a demonstration, we use the generated sample of AMPT with string melting [16, 17]. A partonic phase is implemented in the model and the elliptic flow data from RHIC are well reproduced by the model [18]. For $\mathrm{Au}+\mathrm{Au}$ at $\sqrt{s_{N N}}=200 \mathrm{GeV}$, about 1.6 millions minimum bias events are generated.

\section{AZIMUTHAL DISTRIBUTIONS OF RADIAL MOMENTUM IN AMPT MODEL}

The azimuthal distributions of radial momentum, mean radial momentum, and multiplicity are presented in Fig. 1(a), (b) and (c), respectively. Error is statistical only and smaller than the size of the points. The particles within rapidity range $y \in[-5,5]$ are counted. These cases are kept in all the following figures.

We can see from Fig. 1 that all the observables as a function of azimuthal angle show the anisotropic shape, $\cos (2 \phi)$. It is the same as multiplicity distribution, the biggest anisotropy of mean radial momentum distribution appears in in-plan direction as shown in Fig. 1(b). It indicates that not only the particle density, but also the associated $p_{\mathrm{t}}$ are larger in in-plane direction. It is interesting to see if the data at RHIC show the same character as the model.

In order to compare the anisotropy effects of these three distributions qualitatively, the centrality dependence of the corresponding anisotropic parameter, $v_{2}$, is presented in Fig. 2. The anisotropy parameter $v_{2}$ from different measurements shows the similar centrality dependencies. At each centralities, the anisotropy parameter of multiplicity distribution, $v_{2}(N)$, is larger than that 

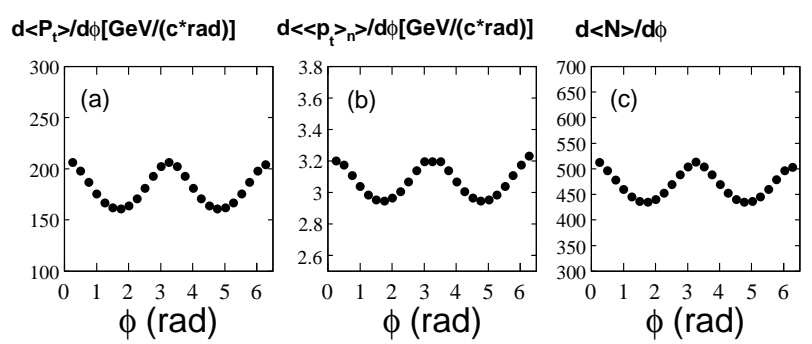

FIG. 1: The azimuthal distributions of radial momentum (a), mean radial momentum (b), and multiplicity (c) for the sample of $\mathrm{Au}+\mathrm{Au}$ collisions at $\sqrt{s_{N N}}=200 \mathrm{GeV}$ generated by AMPT with string melting.

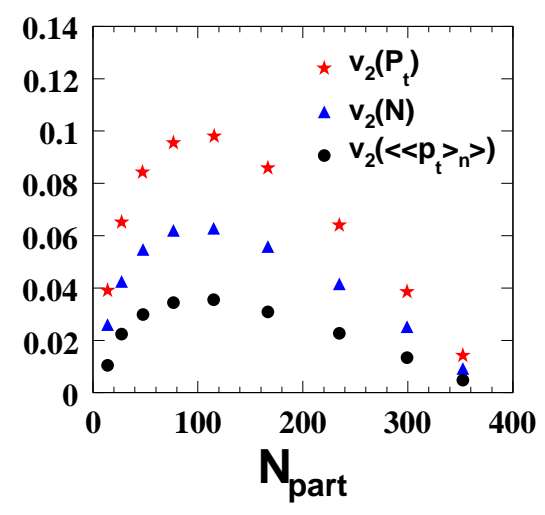

FIG. 2: (Color online)The centrality dependence of elliptic flow parameters deduced from azimuthal distributions of radial momentum (solid red stars), mean radial momentum (solid black cycles), and multiplicity (solid blue triangles) for the sample of $\mathrm{Au}+\mathrm{Au}$ collisions at $\sqrt{s_{N N}}=200 \mathrm{GeV}$ generated by AMPT with string melting.

of the mean radial momentum distribution $v_{2}\left(\left\langle\left\langle p_{t}\right\rangle\right\rangle\right)$. The anisotropy parameter of radial momentum, $v_{2}\left(\left\langle P_{t}\right\rangle\right)$, is the largest one among the three variables. It confirms that the anisotropy of radial momentum distribution including the contributions from a number of particles and their associated transverse momentum. Therefore, the azimuthal distribution of radial momentum gives a full count of anisotropic expansion.

As we know, the anisotropy parameters $v_{2}$ also depend on $p_{\mathrm{t}}$, and it increases with $p_{\mathrm{t}}$ when $p_{\mathrm{t}}<2 \mathrm{GeV} / c[19]$. The $p_{\mathrm{t}}$ dependence of the anisotropy parameters of radial momentum and multiplicity distributions are presented in Fig. 3. The anisotropy parameter increases with $p_{\mathrm{t}}$ when $p_{\mathrm{t}}<2 \mathrm{GeV} / c$, the same as the data shown. We can also see that the $v_{2}$ slightly decreases with $p_{\mathrm{t}}$ when $p_{\mathrm{t}}>2 \mathrm{GeV} / c$, and it may be contributed by the hard components [20]. At a fixed $p_{\mathrm{t}}$ bin, the anisotropy of the radial momentum is almost the same as that of multiplicity. This is because the $p_{\mathrm{t}}$ of all particles in a small given $p_{\mathrm{t}}$ bin are almost the same. The anisotropy of radial

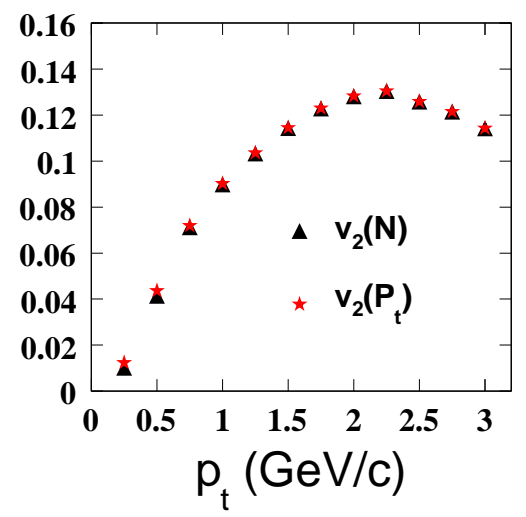

FIG. 3: (Color online) $p_{\mathrm{t}}$ dependence of anisotropic parameter of azimuthal distributions of radial momentum (red solid stars), and multiplicity (black triangles) for the sample of $\mathrm{Au}+\mathrm{Au}$ collisions at $\sqrt{s_{N N}}=200 \mathrm{GeV}$ generated by AMPT with string melting.

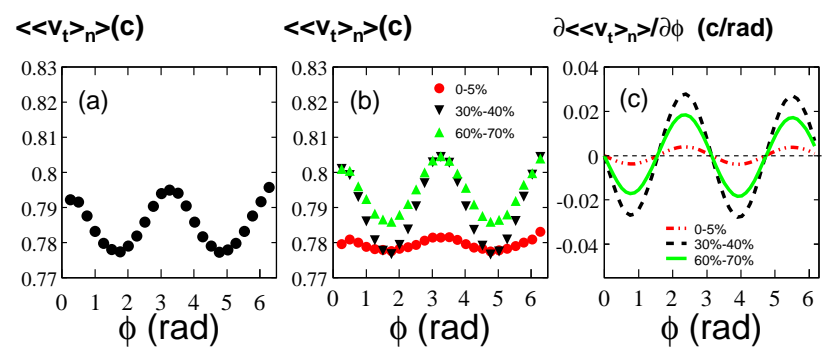

FIG. 4: (Color online) The azimuthal distributions of mean radial velocities of minimum bias sample (a) and the samples of three different centralities (b), and the azimuthal gradients of (b) in (c).

momentum is dominated by that of multiplicity.

\section{AZIMUTHAL DISTRIBUTIONS OF RADIAL VELOCITY IN AMPT MODEL}

The azimuthal distribution of mean radial velocity is presented in Fig. 4(a). It is a period function and can be well fitted by

$$
\left\langle\left\langle V_{t}\right\rangle\right\rangle=V_{0}+V_{a} \cos (2 \phi)
$$

It is the same mode as the flow velocity,

$$
\beta=\beta_{0}+\beta_{a} \cos (2 \phi),
$$

which is usually assumed in blast-wave model in counting the anisotropic expansion [13, 15].

In order to see the contribution of the random thermal motion, the mean radial velocity of final state particles in three typical centralities are presented in Fig. 4(b). In mid-central $(30 \%-40 \%)$ and peripheral $(60 \%-70 \%)$ 
collisions, the mean radial velocities are anisotropy, while it becomes approximately an isotropy constant in central $(0-5 \%)$ collisions. This suggests that the interactions between azimuthal layers are negligible in central collisions, which is consistent with the expectations of viscous hydrodynamics 21, 22]. It also shows that the thermal motions only contribute an isotropic constant to the mean radial velocity.

As we know, for a system with a fixed temperature, the lighter particle has higher thermal velocity. In order to test if the $V_{0}$ is mainly caused by thermal motion, the mean radial velocities of three different particles and their corresponding fitting parameters are presented in Fig. 5 . Indeed, the lightest pion has the highest $V_{0}$, while the heaviest proton has the lowest one.

To see the anisotropy effect alone, we can calculate the gradient of mean radial velocity along the azimuthal direction. In the case, the constant part of the mean radial velocity is canceled. Fig. 4(c) shows the corresponding gradients of Fig. 4(b). In central collisions, it is approximately zero. The amplitudes in mid-central collisions are larger than those in peripheral collisions. These results show that there is almost no gradient of mean radial velocity in central collisions and it becomes the largest in mid-central collisions.

Conventionally, the parameters of flow velocity Eq. (10), $\beta_{0}$ and $\beta_{a}$, are obtained by fitting the spectra of produced particles. Here, we choose the spectra of pion, proton and kaon from AMPT string melting and get, $\beta=0.35+0.04 \cos (2 \phi)$. Due to thermal motion, the $\beta_{0}$ is not directly comparable with $V_{0}$. However, $V_{a} \sim 0.01$ from corresponding mean radial velocity may be a good approximation of flow velocity estimated by blast-wave model, where $\beta_{a} \sim 0.04$.

Certainly, the flow velocities obtained from directly measured radial velocity and from the spectrum fitting based on blast-wave model should be better compared by experimental data sample, where the spectrum is precisely presented. The comparison of these two methods will lead to a better understanding of the flow velocity.

\section{SUMMARY AND CONCLUSIONS}

In the paper, we suggest the studies for azimuthal distributions of radial momentum, mean radial momentum, and mean radial velocity in relativistic heavy heavy ion collisions.

Using the sample of $\mathrm{Au}+\mathrm{Au}$ collisions at $\sqrt{s_{N N}}=$ $200 \mathrm{GeV}$ produced by a multiphase transport model (AMPT), we find that the azimuthal distribution of ra- dial transverse momentum indeed counts the anisotropy of final state particles and their associated transverse momenta. Thus it presents a full description of anisotropic expansion at various centralities. Only in small $p_{\mathrm{t}}$ bin, the azimuthal distribution of radial momentum shows the same anisotropy as that of the multiplicity distribution.

The azimuthal distribution of mean radial velocity is

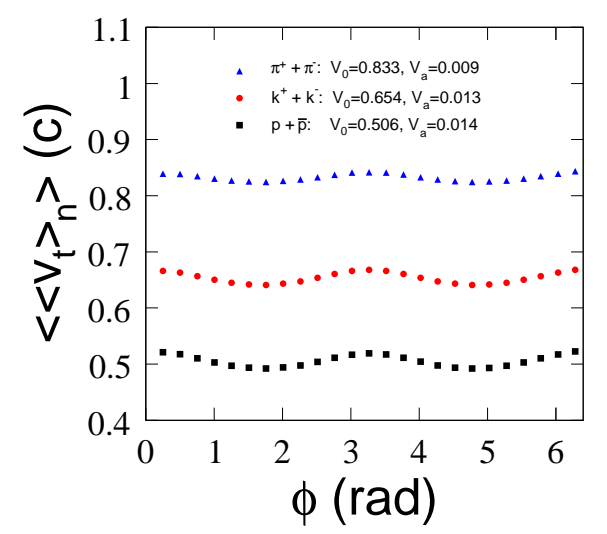

FIG. 5: (Color online)The azimuthal distributions of mean radial velocity of charged pion, charged kaon and (anti)proton. The lines are fitted by Eq. (8), and the corresponding fitted parameters are listed. The errors of the parameters are less than $1 \%$ relative values.

shown to be the same mode as flow velocity which is usually assumed in generalized blast-wave model. Its centrality dependency indicates that thermal motion only contributes an isotropic constant to mean radial velocity. Its particle mass dependency further shows that the mass ordering of isotropic mean radial velocity is the same as thermal motion. The anisotropic mean radial velocity is approximated to flow velocity, which is obtained from fitting the spectrum of corresponding particles based on Blastwave model.

Therefore, it is interesting to measure the azimuthal distributions of radial momentum, mean radial momentum, and mean radial velocity in current relativistic heavy ion collisions.

The first and last authors are grateful for the valuable comments by Dr. Zhangbu Xu. The last author is grateful for the hospitality of BNL STAR group. This work was supported in part by the National Natural Science Foundation of China under Grant No. 10835005 and MOE of China under Grant No. IRT0624 and B08033.
[1] B.Müller, Nucl. Phys. A, 2006, 774 : 433

[2] H. Sorge, Phys. Lett. B, 1997, 402:251; Phys. Rev. Lett. 1997, 78: 2309; Phys. Rev. Lett., 1999, 82: 2048
[3] P. Danielewicz, Phys. Rev. C, 1995, 51:716

[4] S. Voloshin, Y. Zhang, Z. Phys. C , 1996, 70: 665

[5] Sergei A. Voloshin, Arthur M. Poskanzer, and Raimond 
Snellings, arXiv:0809.2949

[6] P. Danielewicz and G. Odyniec, Phys. Lett., 1985, 157B:146

[7] Miklos Gyulassya, Ivan Viteva, Xin-Nian Wang et al Phys.Lett.B , 2002, 526:301-308

[8] P. Huovinen , P. V. Ruuskanen, Ann. Rev. Nucl. Part. Sci., 2006, 56 :163; D. A. Teaney, arXiv: 0905.2433

[9] L.D.Landau, E.M. Lifschitz, Fluid Mechanics, Institute of Physical Problems, U.S.S.R. Academy of Sciences, Volume 6, Course of Theoretical Physics

[10] Y. Oh, Z. Lin, and C. M. Ko, arXiv: 0910.1977

[11] Jajati K. Nayak , Jan-e Alam, Phys. Rev. C , 2009, 80:064906; P. Mohanty, J. K. Nayak, J. Alam et al, arXiv: 0910.4856

[12] J. Y. Ollitrault, Nucl. Phys. A, 1998, 638: 195c

[13] Y. Oh, Z. W. Lin, C. Y. Ko, Phys. Rev. C , 2009, 80:
064902

[14] P. Siemens , J.O. Rasmussen, Phys. Rev. Lett., 1979, 42: 880

[15] P. Huovinen, P.F. Kolb, U. Heinz et al, Phys. Lett. B, 2001, 503: 58

[16] B. Zhang, C.M. Ko, B.A. Li et al, Phys. Rev. C , 2000, 61: 067901

[17] Zi-Wei Lin, Che Ming Ko, Bao-An Li et al, Phys. Rev. C, 2005, 72: 064901

[18] Z.W. Lin and C. M. Ko, Phys. Rev. C , 2002,65: 034904

[19] J. Manninen, F. B., Phys. Rev. C , 2008,77: 054901

[20] R. C. Hwa, arXiv:1009.0506

[21] H. Song, U. W. Heinz, Phys.Rev. C, 2008, 77 :064901

[22] Huichao Song, arXiv: 0908.3656 